\title{
Defining parameters for control of extruding wire by friction process
}

\author{
Nuray Hasan ${ }^{1}$ and Deyan Gradinarov ${ }^{1}$ \\ ${ }^{1}$ Institute of Metal Science, equipment, and technologies "Acad. A. Balevski" with Center for Hydro- and Aerodynamics at the \\ Bulgarian Academy of Sciences
}

\begin{abstract}
Friction stir extrusion is an innovative direct-recycling technology for metal machining chips. During the process a specifically designed rotating tool is plunged into a cylindrical matrix containing the scraps to be recycled. The stirring action of the tool prompts solid bonding related phenomena allowing the back extrusion of a full dense rod. This process results to be particularly relevant because allows the reuse of the scrap without any previous treatment. Experiments have been carried out in order to investigate the influence of the process parameters on the extrudes quality and a numerical model has been developed in order to simulate the evolution of the material flow.
\end{abstract}

\section{Extrusion by friction}

The production of most mechanical component requires machining operation, thus usually implying the cut material to be wasted as scrap during in traditional cutting processes [1]. The recovery of these materials results to be nowadays a crucial challenge in order to obtain both environmental and economic advantages thus leading many researchers and industries to look for innovative and effective recycling technologies. Unfortunately, machining chip is one of the most difficult kinds of scrap to be processed because of some critical characteristics as elevated surface/volume ratio, the presence of oxide layers and different types of contaminants residues i.e. lubricants used during the cutting process. The conventional melting recycling technologies may be applied with many different drawbacks in terms environmental issues (fumes and gas formation), energetic/economic issues, (low efficiency in terms of obtained material and high energetic cost) and technological issues (defectiveness in the final product). In the last years, the recycling by melting of aluminum and magnesium alloys has been deeply investigated by many researchers $[2,3]$ showing that usually the overall recovery rate hardly reaches $50 \%$. All the above-cited issues these conventional technologies to be generally inadequate for the modern industrial needs.

Various products that have been developed from extruded aluminum include rails, mullions, tracks, frames and heat sinks.

Gronostajski and Matuszak [4] first introduced in 1999 the direct conversion method. The metal scraps were separated according to the composition, cleaned, chopped and finally compacted and hot extruded between $500^{\circ} \mathrm{C}$ and $550^{\circ} \mathrm{C}$. The direct chip recycling is a relatively simple technology, providing high recovery efficiency and characterized by lower environmental impact compared to the conventional methods, as showed by Duflou et al. [5].

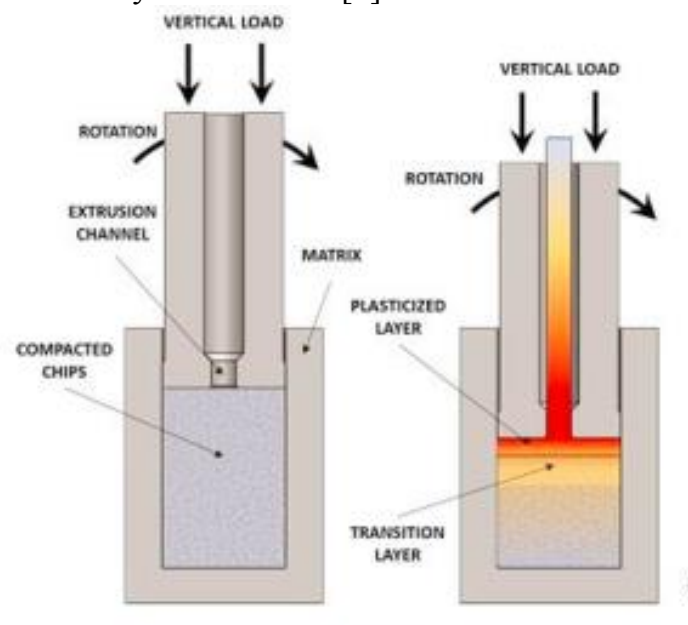

(a)

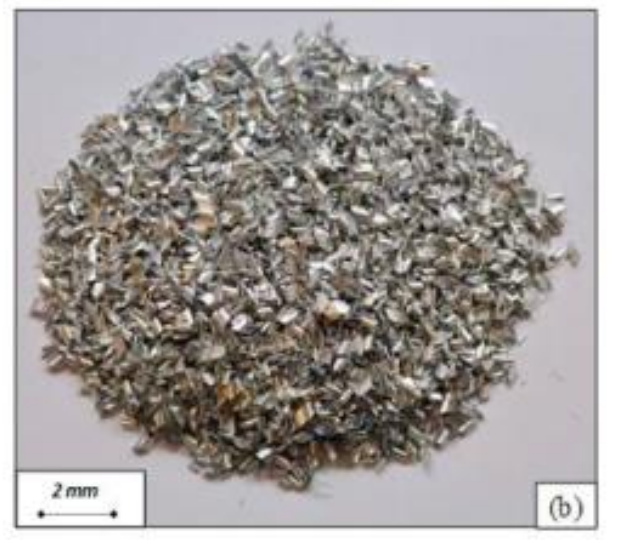

Fig. 1. Sketch of the Friction Stir Extrusion process (a) and AA2024 metal chips used during the experimental campaign (b) $[6]$. 
In 1993 TWI patented a new recycling process to be applied to metal chips, named Friction Stir Extrusion (FSE). This technique belongs to the Friction Stir Processing (FSP) technologies, developed following up the "Friction Stir Welding" (FSW). A rotating tool is used to produce heat and plastic deformation through friction between the tool itself and the chips to be recycled (into a hollow cylindrical matrix) by compacting, stirring and extruding the material. In this way, the recycling process takes place in a unique operation, resulting in significant cost, energy, and labor saving with respect to both conventional method and direct method. For this reason, the FSE technique appears very attractive to industry for the recycling of machining chips. Fig. 1a shows a sketch of the process: the chip closer to the tool, i.e. closer to the heat source, rotates together with the tool and plasticizes due to the combined effect of high temperature and stirring. Moving far from the tool interface, a transition layer is encountered, in which the chip is heated but has not been homogenized as a continuum material. The extrusion starts from the rotating plasticized layer and is influenced by the combined action of tool rotation and force on the tool. At the end of the process, the extruded material returns to room temperature by calm air cooling. After the TWI patent expiration, due to failure to pay maintenance fee in 2002 , only very few papers have been published on the process. In particular, Tang and Reynolds [7], produced AA2050 and AA2195 wires from chips using fixed extrusion force and varying tool rotation. The microstructure of the extruded wires is characterized by small equiaxial grains resulting in good mechanical properties of the wires in terms of microhardness and bend ductility. Some of the authors of this paper have already carried out an experimental campaign on the FSE of AZ31 Mg alloy imposing both extrusion speed [8] and force [9] through the control of rotating tool vertical movement. The obtained results show that the process is feasible and mechanical resistance above $90 \%$ of the base material can be reached. Tool rotation is key process parameter for the effectiveness of the process. With low rotation values, corresponding to low heat input, no extrusion is obtained. On the contrary, the combination of large rotation values and high strain can result in swirl defects compromising the specimen mechanical properties. A complex $3 \mathrm{D}$ helical material flow is generated by the tool action, and distinct areas are observed in the cross section of the extruded parts, with heavily stretched grain in the periphery and recrystallized grain in the center. Although FSE can be considered extremely competitive even compared to the direct method, the real potential of the process has not been still highlighted due to the significant knowledge gap in literature. Analyzing and isolating the effects of each of the different technological parameters on the mechanical properties of the produced rod can be quite difficult. To reduce the amount of the experiments a numerical model able to predict the main fields variable as well as the extrudates quality can represent a useful design tool, usually applied in other Friction Stir process i.e. FSW [10]. Some of the authors of this paper have developed a "single-block" 3D FEM model [9] to simulate most relevant field variables, material flow and embedding a bonding parameter capable of predicting defectiveness. In the last decades, many researchers have investigated the possibility to produce metal matrix composites recurring to innovative technologies, such as friction stirbased technologies. Dolatkhan et al. [11] investigated the effect of process parameter on the fabrication of AA5052/SiC Metal Matrix Composite (MMC) through Friction Stir Processing, while the same technology was applied to AA2024/SiC [12] and Al/Graphene [13]. No paper is known to the authors on the capability of FSE of directly fabricating MMC. In this paper, the results of an experimental campaign on FSE of AA2024 aluminum alloy chips, aimed at the production of MMC within the recycling process by adding $\mathrm{SiC}$ powder to the chips, are presented. Different values of the percentage of the $\mathrm{SiC}$ powder with respect to the chips to be recycled have been studied. Numerical simulation was used to highlight the material flow during the process and identify the process mechanics leading to the formation of big $\mathrm{SiC}$ agglomerates in case of high initial percentage.

\section{Parameters of the process}

Friction Extrusion is a process requiring no external heat source. Heat is generated through friction created by relative rotation of the raw material relative to a die set while under load. As material is plasticized, it is forced to flow through the die. It can be used to form fully consolidated wire, rods, tubes, or other noncircular metal shapes directly from a variety of precursor charges including metal powder, flake, machining waste (chips or swarf) or solid billet.

Schematic illustration of designed setup can be seen in Fig.2. Production of wire from machined aluminum chips includes four main steps:

As it can be seen in Fig.2a, stationary billet chamber was in stationary side of conventional turning machine. It should be mentioned that a conventional turning machine had been utilized in this study to supply required movements for friction stir extrusion die. In second step aluminum chips filled in stationary side of extrusion die and compressed into it (Fig.2b). The third stage is directing rotating die with a load into the stationary billet chamber.

Materials produced by the Friction Extrusion process have a very fine grain structure, which has the potential to provide the following benefits as compared to conventional extruded material: higher fatigue strength; better resistance to corrosion; improved formability.

The structure of the extruded wire depends in the temperature in the billet chamber $\left(T_{\text {in }}\right)$ and the temperature of the point where the wire exits $\left(\mathrm{T}_{\text {out }}\right)-$ cooling temperature. The speed of extruding the wire makes it cool faster of slower and by controlling it we can control the structure of the extruded wire.

For evenly distribution of the melted metaling and alloying components in the billet chamber is crucial to control the angular velocity of the rotating die $(w)$ and 
with this the rotating speed of the die at each pit of its surface $\left(\mathrm{S}_{\mathrm{rD}}(\mathrm{i})\right)$ and the applied external force $(\mathrm{F})$ on the die.
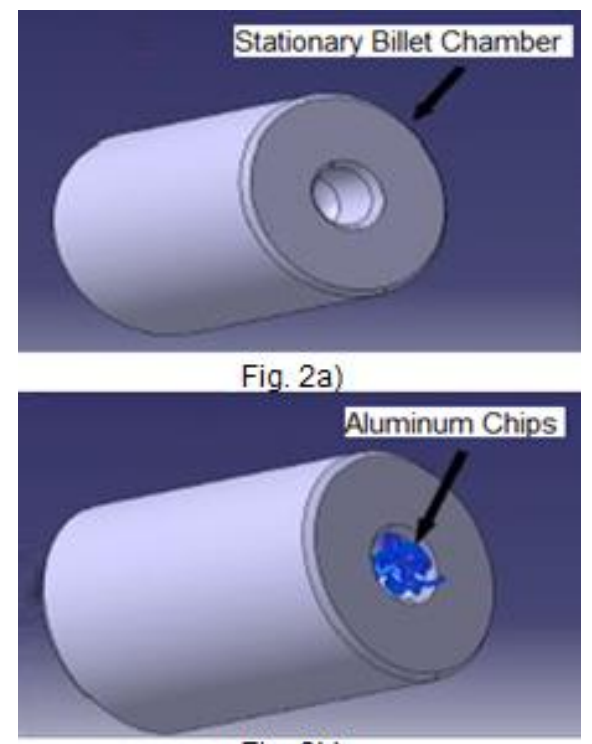

Fig. 2b)

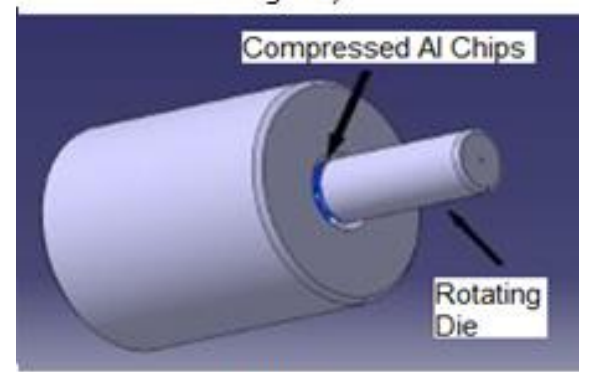

Fig. 2c)

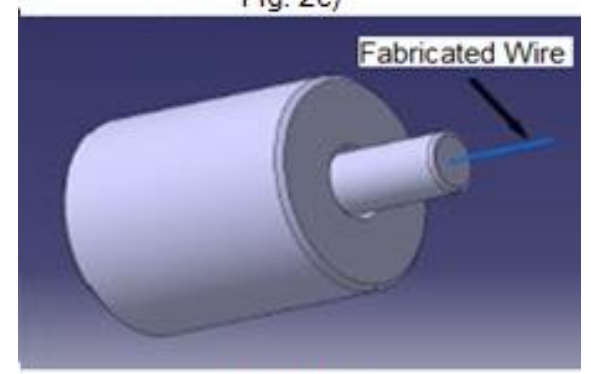

Fig. 2d)

Fig. 2. Schematic of friction stir extrusion process to produce aluminum wires from chips [14].

\section{Conclusions}

- The process of friction stir extrusion is innovative and perspective but not enough studied. In order to be improved through innovative experimental set-up the process have to be studied more thoroughly.

- The process of friction stir extrusion allows easily pulling of wire. The chemical compound of the pulled wire can be easily achieved by adding alloying elements in the chips.

- By controlling the extrusion process parameters, we can acquire needed structure of the wire.

- By studying the influence of the parameters of the process upon characteristics of the extruded wire we can refine the process and make it more efficient and effective.

\section{Reference}

1. W.M. Thomas, E.D. Nicholas, S.B. Jones, US Patent \# 5,262,123 (1993)

2. J. Gronostajski, A. Matuszak, The recycling of metals by plastic deformation: an example of recycling of aluminum and its alloys chips, PT, 9293 (1999)

3. F. Jovane, H. Yoshikawa, L. Alting, C.R. Boër, E. Westkamper, D. Williams, M. Tseng, G. Seliger, A.M. Paci, The incoming global technological and industrial revolution towards competitive sustainable manufacturing, MT, 641-659 (2008)

4. T.G. Gutowski, J.M. Allwood, C. Herrmann, S. Sahni, A global assessment of manufacturing: Economic development, energy use, carbon emissions, and the potential for energy efficiency and materials recycling, ARERs, 81-106 (2013)

5. G. Hanko, H. Antrekowitsch, P. Ebner, Recycling automotive magnesium scrap, JMMMS, 51-54 (2002)

6. M. Samuel, A new technique for recycling aluminium scrap. JMPT, 117-124 (2003)

7. J. Gronostajski, A. Matuszak, Recycling of metals by plastic deformation: an example of recycling of aluminium and its alloys chips, JMPT, 35-41 (1999)

8. J.R. Duflou, A.E. Tekkaya, M. Haase, T. Welo, K. Vanmeensel, K. Kellens, W. Dewulf, D. Paraskevas, Environmental assessment of solidstate recycling routes for aluminium alloys: Can solid state processes significantly reduce the environmental impact of aluminium recycling $\mathrm{MT}$, 37-40 (2015)

9. W. Tang, A.P. Reynolds, Production of wire via friction extrusion of aluminum alloy machining chips, JMPT 210(15), 2231-2237 (2010)

10. G. Buffa, D. Campanella, L. Fratini, F. Micari, AZ31 magnesium alloy recycling through friction stir extrusion process, IJMF (In press) (2015)

11. D. Baffari, G. Buffa, L. Fratini, A numerical model for Wire integrity prediction in Friction Stir Extrusion of magnesium alloys, JMPT 247, 1-10 (2017)

12. C. McAuliffe, R. Karkkainen, C. Yen, H. Waisman, Numerical modelling of friction stir welded aluminum joints under high rate loading, FEA, 8-18 (2014)

13. D. Ghanbari, M. Kasiri Asgharani, K. Amini, Investigating the Effect of Passes Number on Microstructural and Mechanical Properties of the A12024/SiC Composite Produced by Friction Stir Processing, Mechanics 21(6) (2016).

14. W. Tang, A.P. Reynolds, Production of wire via friction extrusion of aluminum alloy machining chips, JMPT (2010) 